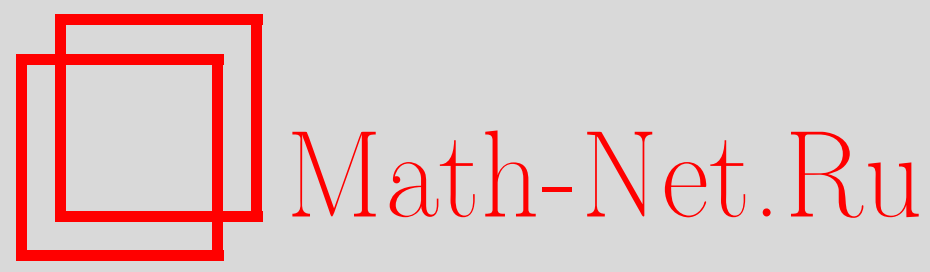

А. Д. Иоффе, В. М. Тихомиров, Несколько замечаний о вариационных принципах, Матем. заметки, 1997, том 61, выпуск 2, 305-311

DOI: https://doi.org/10.4213/mzm2839

Использование Общероссийского математического портала Math-Net.Ru подразумевает, что вы прочитали и согласны с пользовательским соглашением http://www . mathnet.ru/rus/agreement

Параметры загрузки:

IP : 54.237 .59 .107

26 апреля 2023 г., 15:42:11

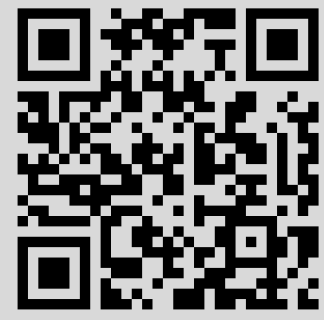




\section{НЕСКОЛЬКО ЗАМЕЧАНИЙ О ВАРИАЦИОННЫХ ПРИНЦИПАХ}

\section{А. Д. Иоффе, В.М. Тихомиров}

Введение. Прошло двадцать лет со дня появления вариационного принципа Экланда [1], оказавшегося сильным и удобным инструментом нелинейного анализа. Вместе с двумя его наиболее известными модификациями, предложенньми Борвейном и Прайсом [2] и ДеВиллем [3] (см. также [5]), принцип Экланда лег в основу новой техники, эффективно работающей в разнообразных ситуациях, позволившей доказать много новых и усилить ряд известных результатов в разнообразных областях анализа, геометрии, теории экстремума, теории Люстерника-Шнирельмана, теории Гамильтона-Якоби и др.

Вариационными приниипами назьвают группу результатов, показьвающих, что полунепрерьвную снизу и ограниченную снизу функцию на полном метрическом пространстве можно произвольно мало возмутить так, что возмущенная функция будет иметь абсолютный (и даже строгий) минимум.

Непрерывную функцию $\varphi_{x, \alpha}(\cdot)(\alpha>0)$, заданную на метрическом пространстве $(X, d)$ назовем холм-функиией (от bump-function), сосредоточенной на $B(x, \alpha):=$ $\{u \in X \mid d(x, u) \leqslant \alpha\}$, если $\varphi_{x, \alpha}(x)=1$. Она ограничена, неотрицательна на $B(x, \alpha)$ и неположительна вне этого шара.

Вот формулировки вариационных принципов Экланда, Борвейна-Прайса и ДеВилля.

ПРИНЦИП ЭКЛАНДа [1]. Пусть $(X, d)$ - полное метрическое пространство, $f$ полунепрерывная снизу и ограниченная снизу функиия на $X$, причем $f(w) \leqslant \inf f+\varepsilon$.

Тогда для любого $\lambda>0$ существует әлемент $x_{*}$, для которого

(a) $f\left(x_{*}\right) \leqslant f(w)$,

(b) $d\left(w, x_{*}\right) \leqslant \lambda$,

(c) $f(x)+\frac{\varepsilon}{\lambda} d\left(x, x_{*}\right)>f\left(x_{*}\right) \forall x \neq x_{*}\left(\right.$ m.е. возмущенная функция $f(\cdot)+\frac{\varepsilon}{\lambda} d\left(\cdot, x_{*}\right)$ достигает строгого абсолютного минимума в точке $x_{*}$ ).

ПРИНЦИП БОРВЕЙНА-ПРАЙСА [2]. Если в предыдущих условия $X$ - банахово пространство, то $\forall \lambda>0, p \geqslant 1$ и любой последовательности

$$
\left\{\mu_{n}\right\}_{n \in \mathbb{N}}, \quad \sum_{n \in \mathbb{N}} \mu_{n}=1
$$

существует последовательность $\left\{x_{n}\right\}_{n \in \mathbb{N}} \subset X$, сходящаяся $\kappa x_{*}$, и при этом

(a) $f\left(x_{*}\right) \leqslant f(w)$

(b) $d\left(w, x_{*}\right) \leqslant \lambda$,

(c) функиия

$$
f(\cdot)+\varepsilon \lambda^{-p} \sum_{n \in \mathbb{N}} \mu_{n}\left\|\cdot-x_{n}\right\|^{p}
$$

достигает абсолютного минимума в $x_{*}$. 
Принцип ДЕВилля [3], [4] (см. также [5]). Пусть X - банахово пространство, $в$ котором существует некоторая дифференцируемая по Фреше холм-функиия $\varphi(\cdot)$. Пусть $\mathscr{F}$ - банахово пространство ограниченных на $X$ функиий с топологией не слабее топологии равномерной сходимости, содержащее холм-функиии вида $\varphi(\lambda \cdot+a)(\lambda \in \mathbb{R}, a \in X)$.

Тогда множество функиий $g \in \mathscr{F}$, обладающих тем свойством, что $f+g$ достигают строгого абсолютного минимума в некоторой точке, есть плотное $G_{\delta}$-подмножество $\mathscr{F}$.

Вопреки распространенному мнению все три принципа являются независимьми и не следуют один из другого. Дело не только в разных требованиях на пространство: ниже мы приводим метрические обобщения принципов Борвейна-Прайса и ДеВилля. Принцип Борвейна-Прайса (в отличие от принципа Экланда) не гарантирует, что возмущенная функция имеет строгий минимум, а принцип ДеВилля (в отличие от первых двух) не позволяет локализовать точку абсолютного минимума с той же точностью.

Естественно попытаться понять единую суть всех трех вариационных принципов и найти объединяющую их формулировку. В данной статье мы решаем эту задачу почти полностью. Основной результат, доказываемый далее - это "конструктивный" вариационный принцип, обобщающий принцип Борвейна-Прайса. Из него следует (в существенном) и принцип Экланда.

Само же наше доказательство (как и доказательство теоремы 1 ) фактически навеяно основньм мотивом доказательства принципа ДеВилля - холм-функциями.

Мы показываем связи конструктивного вариационного принципа с принципами Экланда и Борвейна-Прайса, доказываем метрический вариант принципа ДеВилля и в заключение обсуждаем связи между итерационными принципами Ньютона-Люстерника-Грейвса и вариационными принципами. Существует большая группа теорем (типа теорем о накрытиях и регулярности), к доказательству которых применялись и итерационные методы и вариационные принципы. В заключение статьи мы объясняем, что теоремы существования, получаемые итерационньми процедурами, следуют также из принципа Экланда.

1. Конструктивный вариационный принцип. Мыначнем с формулировки простого результата, чье доказательство на наш взгляд весьма красноречиво и просто объясняет то, что лежит в основе вариационных принципов.

Назовем семейство холм-функций $\Phi$ полным, если для любого $x \in X$ и любого $\alpha>0$ множество $\Phi$ содержит холм-функцию $\varphi_{x, \alpha}(\cdot)$, сосредоточенную на $B(x, \alpha)$ такую, что для любого фиксированного $x$ эти функции непрерьвны по $\alpha$ равномерно на каждом шаре с центром $x$ (это означает, что $\varphi_{x, \alpha^{\prime}}(u) \rightarrow \varphi_{x, \alpha}(u)$ равномерно на шаре), и семейство функций $\left\{\varphi_{x, \alpha}(\cdot)\right\}$, где $\{(x, \alpha)\}$ пробегают ограниченное множество в $X \times \mathbb{R}_{+}$, равномерно ограничено на любом ограниченном множестве $X$.

Очевидный пример такого семейства $\varphi_{x, \alpha}(\cdot)=1-\alpha^{-1} d(\cdot, x)$.

Теорема 1. Пусть $(X, d)$ - полное метрическое пространство, $\Phi$ - полное семейство холм-функиий на $X, f$ - полунепрерывная снизу на $X$, неотрицательная функиия и при этом существуют $w \in X$ и $\varepsilon>0$ такие, что $f(w) \leqslant \varepsilon$.

Тогда для любого $\lambda>0$ существует число $0<\alpha_{*}<1$ такое, что для любых последовательностей $\left\{\alpha_{n}\right\}_{n \in \mathbb{Z}_{+}},\left\{\beta_{n}\right\}_{n \in \mathbb{N}}$ положительных чисел, удовлетворяющих 
условиям

$$
\alpha_{0} \leqslant \lambda \alpha_{*}, \quad \sum_{n=0}^{\infty} \alpha_{n} \leqslant \lambda, \quad \sum_{n=1}^{\infty} \beta_{n} \leqslant 1
$$

существует последовательность $\left\{x_{n}\right\}_{n \in \mathbb{Z}_{+}}, x_{n} \in X$, сходящаяся $\kappa x_{*} \in X$, такая, что

(a) $d\left(x_{n}, w\right) \leqslant \lambda$

(b) $f\left(x_{n}\right) \leqslant f(w)$,

(c) существует последовательность $\left\{\gamma_{n}\right\}_{n \in \mathbb{Z}_{+}}$такая, что $0 \leqslant \gamma_{n} \leqslant \beta_{n} \forall n \in \mathbb{N}$, и при этом функиия

$$
g(\cdot)=f(\cdot)-\varepsilon \sum_{n \in \mathbb{Z}_{+}} \gamma_{n} \varphi_{n}(\cdot)
$$

достигает абсолютного минимума в точке $x_{*}\left(\right.$ де мы положили $\varphi_{n}(\cdot)=$ $\left.\varphi_{x_{n}, \alpha_{n}}(\cdot)\right)$.

ДокАЗАТЕЛЬСТво. Достаточно рассмотреть случай $\lambda=1$, которого всегда можно достичь, заменяя $d(\cdot, \cdot)$ на $\lambda d(\cdot, \cdot)$. Положим $x_{0}:=w, \varphi_{0}(\cdot):=\varphi_{x_{0}, \alpha_{0}}(\cdot)$,

$$
\gamma_{0}:=\sup \left\{\gamma \geqslant 0 \mid f(u)-\varepsilon \gamma \varphi_{0}(u) \geqslant 0 \forall u\right\}
$$

Если $\gamma_{0}=1$, то, положив для $n \geqslant 1 \gamma_{n}=0$, получаем утверждение теоремы.

Пусть $\gamma_{0}<1$. Функция $f_{1}:=f-\varepsilon \gamma_{0} \varphi_{0}$, очевидно, также неотрицательна, полунепрерьвна снизу и нижняя грань этой функции на шаре $B\left(x_{0}, \alpha_{0}\right)$ равна нулю. Если эта нижняя грань достигается в некоторой точке $x_{*}$, то снова получается утверждение теоремы. Если же нижняя грань не достигается, то найдется точка $x_{1} \in B\left(x_{0}, \alpha_{0}\right)$, в которой $f_{1}\left(x_{1}\right) \leqslant \varepsilon \beta_{1}$, и мы можем повторить всю конструкцию, заменив $f$ на $f_{1}, \varepsilon$ на $\varepsilon \beta_{1}, \alpha_{0}$ на $\alpha_{1}, w$ на $x_{1}$ и $\varphi_{0}$ на $\varphi_{1}$. В итоге получим $x_{2} \in B\left(x_{1}, \alpha_{1}\right)$, затем $x_{3} \in B\left(x_{2}, \alpha_{2}\right)$ и т.д. Последовательность $\left\{x_{n}\right\}$ - последовательность Коши. В силу полноты $X$ она сходится к некоторому элементу $x_{*}$, а так как по построению $d\left(x_{n}, x_{n+1}\right) \leqslant \alpha_{n}$, получаем утверждение (а) теоремы. Из того, что $f_{n}\left(x_{n}\right) \leqslant \varepsilon \beta_{n}$ и функция $f$ полунепрерывна снизу, вытекает, что $g\left(x_{*}\right)=0$, а вместе с тем $g$ по построению неотрицательна. Значит, выполнены утверждения (b) и (c) теоремы.

Несколько более сложные рассуждения, чем в теореме 1 позволяют доказать следующий основной результат.

Зафиксировав $w$, положим

$$
r_{\Phi}:=\sup \left\{\varphi_{x, \alpha}(u) \mid u \in B(w, 1), x \in B(w, 1), \alpha \leqslant 1\right\}
$$


Теорема 2. Пусть $(X, d)$ - полное метрическое пространство, $f$ - полунепрерывная снизу, ограниченная снизу на $X$ функиия. Пусть $\Phi$ - полное семейство холм-функиий на $X$ и $\varepsilon>0$ таковы, что $f(w) \leqslant \inf f+\varepsilon$. Тогда для любого $\lambda>0$

- либо функиия $f-\varphi_{w, \lambda}$ достигает абсолютного минимума в $w$,

- либо существуют числа $1>\alpha_{*}>\beta_{*}>0$ такие, что для любых последовательностей $\left\{\alpha_{n}\right\},\left\{\beta_{n}\right\}$ положительных чисел, удовлетворяющих условияM

$$
\alpha_{0} \leqslant \lambda \alpha_{*}, \quad \beta_{0} \leqslant \beta_{*}, \quad \sum_{n=0}^{\infty} \alpha_{n} \leqslant \lambda, \quad \sum_{n=0}^{\infty} \beta_{n} \leqslant 1,
$$

существуют $x_{*} \in X u$ сходящаяся $\kappa x_{*}$ последовательность $\left\{x_{n}\right\} \in X$ такие, что

(a) $d\left(x_{*}, w\right) \leqslant \lambda$

(b) $f\left(x_{*}\right) \leqslant f(w)+\varepsilon\left(r_{\Phi}-1\right)$,

(c) функиия

$$
g(\cdot)=f(\cdot)-\varepsilon \sum_{n=0}^{\infty} \beta_{n} \varphi_{n}(\cdot)
$$

достигает абсолютного минимума в точке $x_{*}$ (где мы снова положили $\left.\varphi_{n}(\cdot)=\varphi_{x_{n}, \alpha_{n}}(\cdot)\right)$.

СлЕДСТВИЕ 1. В предположениях теоремы для любого $\lambda>0$ up 1 существует $\mu_{*} \in[0,1]$ такое, что для любой последовательности $\left\{\mu_{n}\right\}$ полохсительных чисел таких, что

$$
\mu_{*} \leqslant \sum_{n=0}^{\infty} \mu_{n} \leqslant 1
$$

существуют $x_{*}$ и последовательность $\left\{u_{n}\right\}$, сходящ, аяся $\kappa x_{*}$ такие, что

(a) $d\left(u_{n}, w\right) \leqslant \lambda$

(b) $f\left(x_{*}\right) \leqslant f(w)$,

(c) функиия

$$
\overline{g(\cdot)}=f(\cdot)+\varepsilon \lambda^{-p} \sum_{n=0}^{\infty} \mu_{n} d^{p}\left(\cdot, u_{n}\right)
$$

достигает абсолютного минимума в точке $x_{*}$.

Более того, если $f(\cdot)+\varepsilon \lambda^{-p}\left(d^{p}(\cdot, w)-1\right)$ не достигает абсолютного минимума в $w$, тогда $\mu_{*}$ можнно выбрать строго меньшим, чем единица.

ДоказАтЕльство. Функции $\varphi_{x, \alpha}(\cdot)=1-\alpha^{-p} d^{p}(\cdot, x), x \in X, \alpha>0$, образуют полное семейство холм-функций с $r_{\Phi}=1$. Если $f-\varepsilon \lambda^{-p} \varphi_{w, 1}$ достигает абсолютного минимума в $w$, мы получим (a)-(c), положив $u_{n}=w, \mu_{n}=1$ для всех $n$. В ином случае выберем $1>\alpha_{*} \gg 0$ в соответствии с теоремой. Разумеется, можно выбрать их так, чтобы $\alpha_{*}^{p}>\beta_{*}$. Выберем $\mu_{*} \in\left(\beta_{*} \alpha_{*}^{-p}, 1\right)$ и последовательность $\left\{\mu_{n}\right\}$, удовлетворяющую условиям. Пусть, далее, число $N$ выбрано столь большим, чтобы

$$
\sum_{n=0}^{N} \mu_{n} \leqslant \beta_{*} \alpha_{*}^{-p}
$$


Выбрав последовательность $\left\{\alpha_{n}\right\}$ положительных чисел так, чтобы $\alpha_{0}=\lambda \alpha_{*}$ и $\sum \alpha_{n} \leqslant 1$, положим

$$
\beta_{0}=\alpha_{0}^{p} \sum_{n=0}^{N} \mu_{n}, \quad \beta_{n}=\alpha_{n}^{p} \sum_{n=0}^{N} \mu_{N+n} .
$$

Теперь можно применить теорему для этих $\alpha_{n}$ и $\beta_{n}$ и найти $x_{*}$ и последовательность $x_{n}$, сходящуюся к $x_{*}$ и удовлетворяющую свойствам (a)-(c) теоремы. Если теперь положить $u_{n}$ равным $x_{0}$ при $n=0, \ldots, N$ и $x_{N}-n$ при $n>N$, мы получим, что соотношения (a)-(b) тривиально выполняются для $u_{n}$, а из соответствуюшей части теоремы (с учетом того, что $r_{\Phi}=1$ ) почти автоматически выводится и $(\mathrm{c})$.

Следствие 1 представляет собой метрическое обобщение принципа Борвейна-Прайса. Но в одном отношении оно идет дальше: заключительное утверждение следствия (определяемая им свобода выбора $\mu_{n}$ ) есть связующее звено между конструктивньп принципом и принципом Экланда.

Действительно, утверждения принципа Экланда (c '<' замененньг на ' $\leqslant$ ') следуют из следствия 1 сразу: достаточно взять $\varphi_{x, \alpha}(\cdot)=1-\alpha^{-1} d(x, \cdot)$.

Если функция $f+\varepsilon \lambda^{-1} d(\cdot, w)$ не достигает абсолютного минимума в $w$, утверждение (c) также непосредственно вытекает из следствия 1. И лишь если эта функция достигает абсолютного минимума в точке $w$, в этом единственном случае строгое неравенство в (c) не вытекает непосредственно из следствия 1. Здесь требуется дополнительное рассуждение, являющееся незначительньм упрощением доказательства самого принципа Экланда, и мы его не приводим.

2. Обобщение принципа ДеВилля на метрические пространства. Конструктивньй принцип предоставляет большую свободу в выборе возмущения функции $f$, но все же "не дотягивает" до доказательства массивности множества "хороших" возмущающих функций, обеспечиваемого принципом ДеВилля. Нижеследующая теорема обобщает этот принцип на метрические пространства в полном объеме. Доказательство является лишь незначительной модификацией доказательства из [3].

Теорема 3. Пусть $(X, d)$ - полное метрическое пространство и $\mathscr{F}$ - банахово пространство ограниченных непрерывных функиий на $X$ таких, что

(а) для любого $g \in \mathscr{F}$ имеет место неравенство

$$
\|g\|_{\mathscr{F}} \geqslant\|g\|_{\infty}=\sup _{x \in X}|g(x)|
$$

(b) существует полное семейство $\Phi$ холм-функиий на $X$, принадлехсащих $\mathscr{F}$. Пусть, далее, $f$ - собственная, полунепрерывная снизу на $X$ функиия, ограниченная снизу. Тогда мнохсество функиий $g \in \mathscr{F}$, обладающих тем свойством, что $f+g$ достигают абсолютного минимума в одной точке, есть плотное $G_{\delta}$-подмножество $\mathscr{F}$.

ДОКАЗАТЕЛЬСТво. Для любого $n$ множество

$$
U_{n}=\left\{g \in \mathscr{F} \mid \exists z:(f+g)(z)<\inf \left\{(f+g)(x) \mid d(x, z) \geqslant \frac{1}{n}\right\}\right\}
$$


открыто в силу (а). Легко показывается с помошью (b), что $U_{n}$ плотно в $\mathscr{F}$.

Отсюда следует, что $G=\bigcap U_{n}-$ плотное $G_{\delta}$-подмножество $\mathscr{F}$, и для любого $g \in G$ существует последовательность $x_{n}$ такая, что

$$
(f+g)\left(x_{n}\right)<\inf \left\{(f+g)(x) \mid d\left(x, x_{n}\right) \geqslant \frac{1}{n}\right\} .
$$

Ясно, что для $m>n$ мы будем иметь $d\left(x_{m}, x_{n}\right)<1 / n$, ибо иначе удовлетворялись бы неравенства $(f+g)\left(x_{m}\right)>(f+g)\left(x_{n}\right)$ и $\left.(f+g)\left(x_{m}\right)<(f+g)\left(x_{n}\right)\right)$. Значит, $\left\{x_{n}\right\}-$ последовательность Коши, сходящаяся к некоторому $x_{*}$. Но тогда для любого $x \neq x_{*}$ получим, взяв $\varepsilon$ и $m$ так, чтобы удовлетворялось неравенство $\frac{1}{2} d\left(x, x_{*}\right)>\varepsilon>1 / m$,

$$
\begin{aligned}
(f+g)(x) & \leqslant \lim _{n \rightarrow \infty}(f+g)\left(x_{n}\right) \leqslant(f+g)\left(x_{m}\right) \\
& <\inf \left\{(f+g)(u) \mid d\left(u, x_{m}\right) \geqslant \frac{1}{m}\right\} \leqslant(f+g)(x) .
\end{aligned}
$$

СлЕДСтвИЕ. В допущениях теоремы 2 для любой последовательности $\left\{f_{n}\right\}$ собственных полунепрерывных снизу, ограниченных снизу функиий и для любого $\varepsilon>0$ существует функиия $g \in \mathscr{F}$ с нормой, меньшей чем $\varepsilon$, такая, что для любого $n$ функиия $f_{n}+g$ достигает строгого глобального минимума на $X$.

\section{3. Итерационные принципы Ньютона-Люстерника-Грейвса и принцип Экланда.}

ТЕОрема 4. Пусть $\left(X, d_{X}\right)$ - полное метрическое, а $\left(Y, d_{Y}\right)$ - метрическое пространство, в которых заданы точки $x_{0} \in X, y \in Y, F$ - замкнутое многозначное отображсение из $X$ в $Y, r>0 u M: B_{X}\left(x_{0}, r\right) \rightarrow X$ - отобрахсение, обладающее для некоторого положительного $\theta<1$ следующими свойствами: $d_{Y}\left(y, F\left(x_{0}\right)\right) \leqslant(1-\theta) r$ и либо

(a) $d_{X}(x, M(x)) \leqslant d_{Y}(y, F(x)), d(y, F(M(x))) \leqslant \theta d_{X}(x, M(x)) \quad \forall x \in B_{X}\left(x_{0}, r\right)$, либо

(b) $d_{X}(x, M(x)) \leqslant d_{Y}(y, F(x)), d_{Y}(y, F(M(x))) \leqslant \theta d_{Y}(y, F(x)) \quad \forall x \in B_{X}\left(x_{0}, r\right)$. Тогда включение $y \in F(x)$ имеет решение $x_{*}$, более того, $x_{*}$ есть предел последовательности $\left\{x_{k}\right\}$, получаемой простой итерачией отображения $M$ : $x_{k+1}=M\left(x_{k}\right), k \in \mathbb{Z}$, начиная $с$ точки $x_{0}$.

Доказательство этой теоремы очень просто, и мы его не приводим. Сам по себе общий итеративньй принцип решения уравнений восходит, по-видимому, к Ньютону. В бесконечномерном случае подобные конструкции применяли Люстерник, Грейвс (для доказательства вариантов теоремы о неявной функции), Канторович и многие другие. Многие подобные результаты непосредственно следуют из теоремы 4 (в частности, теорема Дмитрука-Милютина-Осмоловского из [4]). Покажем, что сама теорема 4 является следствием принципа Экланда.

Действительно, заменив $d_{X}$ и $d_{Y}$ на $r^{-1} d_{X}$ и $r^{-1} d_{Y}$ соответственно, сведем дело к случаю $r=1$. Пусть $w=x_{0}$. Рассмотрим функцию $f(x)=d_{Y}(y, F(x))$. Из замкнутости $F$ следует, что $f$ полунепрерывна снизу. Очевидно, что она неотрищательна и 
$f(w) \leqslant 1-\theta$. В силу принципа Экланда, существует $x_{*}$ с условием $d_{X}\left(x_{*}, w\right) \leqslant 1$ такое, что $f(x)+(1-\theta) d_{X}\left(x, x_{*}\right)>f\left(x_{*}\right)$, если $x \neq x_{*}$. Рассмотрим точку $x=M\left(x_{*}\right)$. Из вторых неравенств в условиях (a), (b) теоремы 4 вытекает, что $x_{*}=M\left(x_{*}\right)$. Тогда

$$
\begin{aligned}
d_{Y}\left(y, F\left(x_{*}\right)\right) & <d_{Y}\left(y, F\left(M\left(x_{*}\right)\right)\right)+(1-\theta) d_{X}\left(x_{*}, M\left(x_{*}\right)\right) \\
& \leqslant d_{Y}\left(y, F\left(M\left(x_{*}\right)\right)\right)+(1-\theta) d_{Y}\left(y, F\left(x_{*}\right)\right) .
\end{aligned}
$$

В случае (а) имеем $d_{Y}\left(y, M\left(F x_{*}\right)\right) \leqslant d_{Y}\left(y, F\left(x_{*}\right)\right)$, в случае (b) мы получаем то же неравенство из второго неравенства в (b). В обоих случаях (в предположении $y \notin F\left(x_{*}\right)$ ) приходим к противоречию: $d_{Y}\left(y, F\left(x_{*}\right)\right)<d_{Y}\left(y, F\left(x_{*}\right)\right)$.

Мы получили основное утверждение теоремы 4 (впрочем, не столь конструктивно), а заодно убедились в важности строгого неравенства в утверждении (c) принщипа Экланда.

Московский государственный университет

Поступило им. М. В. Ломоносова

09.10 .96

\section{СПИСОК ЦИТИРОВАННОЙ ЛИТЕРАТУРЫ}

1. Ekeland I. // J. Math. Anal. Appl. 1974. V. 47. P. 324-325. 2. Borwein J. M., Preiss D. // Trans. Amer. Math. Soc. 1987. V. 303. P. 517-527. 3. DeVille R. Nouveaux principes variationnelles // Sem. Init. 'a l'Analyse / ed. G. Choquet, G. Godefroy, M. Rogalsky, J. Saint-Rayvond. 30-e Anne’e 1990/91 № 21. 4. Дмитрук А. В., Милютин А. А., Осмоловский Н. П. // УМН. 1980. T. 35. №6. C. 11-46. 5. Ekeland I. // Bull. Amer. Math. Soc. 1979. P. 433-479. 